\title{
Serious Illness Conversation-Evaluation Exercise: A Novel Assessment Tool for Residents Leading Serious Illness Conversations
}

\author{
Jenny J. Ko, MD, FRCPC, ${ }^{1, *}$ Mark S. Ballard, MD, FRCPC, ${ }^{2}$ Tamara Shenkier, MDCM, FRCPC, ${ }^{3}$ \\ Jessica Simon, MB, ChB, FRCPC, ${ }^{4}$ Amanda Roze des Ordons, MD, FRCPC, MMEd, ${ }^{4}$ \\ Gillian Fyles, MD, CCFP(PC), ${ }^{5}$ Shilo Lefresne, MD, FRCPC, ${ }^{6}$ Philippa Hawley, BMed, FRCPC, \\ Charlie Chen, MD, CCFP(PC), MEd, ${ }^{4}$ Michael McKenzie, MD, FRCPC, ${ }^{6}$ Isabella Ghement, PhD, ${ }^{8}$ \\ Justin J. Sanders, MD, MSc, Rachelle Bernacki, MD, MSc, and Scott Jones, RN, BScN, BEd ${ }^{10}$
}

\begin{abstract}
Background/Objectives: The serious illness conversation (SIC) is an evidence-based framework for conversations with patients about a serious illness diagnosis. The objective of our study was to develop and validate a novel tool, the SIC-evaluation exercise (SIC-Ex), to facilitate assessment of resident-led conversations with oncology patients.

Design: We developed the SIC-Ex based on SIC and on the Royal College of Canada Medical Oncology milestones. Seven resident trainees and 10 evaluators were recruited. Each trainee conducted an SIC with a patient, which was videotaped. The evaluators watched the videos and evaluated each trainee by using the novel SIC-Ex and the reference Calgary-Cambridge guide (CCG) at months zero and three. We used Kane's validity framework to assess validity.

Results: Intra-class correlation using average SIC-Ex scores showed a moderate level of inter-evaluator agreement (range 0.523-0.822). Most evaluators rated a particular resident similar to the group average, except for one to two evaluator outliers in each domain. Test-retest reliability showed a moderate level of consistency among SIC-Ex scores at months zero and three. Global rating at zero and three months showed fair to good/very good inter-evaluator correlation. Pearson correlation coefficients comparing total SIC-Ex and CCG scores were high for most evaluators. Self-scores by trainees did not correlate well with scores by evaluators.

Conclusions: SIC-EX is the first assessment tool that provides evidence for incorporating the SIG guide framework for evaluation of resident competence. SIC-Ex is conceptually related to, but more specific than, CCG in evaluating serious illness conversation skills.
\end{abstract}

Keywords: advance care planning; resident education; serious illness conversation

\footnotetext{
'Department of Medical Oncology, University of British Columbia, BC Cancer-Abbotsford, Abbotsford, British Columbia, Canada.

${ }^{2}$ Department of Internal Medicine, Chilliwack General Hospital, Chilliwack, British Columbia, Canada.

${ }^{3}$ Department of Medical Oncology, ${ }^{6}$ Department of Radiation Oncology, ${ }^{7}$ Pain and Symptom Management/Palliative Care Program, BC Cancer-Vancouver, Vancouver, British Columbia, Canada.

${ }^{4}$ Department of Oncology, University of Calgary, Calgary, Alberta, Canada.

${ }^{5} \mathrm{BC}$ Centre for Palliative Care, Vancouver, British Columbia, Canada.

${ }^{8}$ Ghement Statistics, Inc., Richmond, British Columbia, Canada.

${ }^{9}$ Ariadne Labs, Dana-Farber Cancer Institute, Boston, Massachusetts, USA.

${ }^{10}$ Vancouver Coastal Health, Vancouver, British Columbia, Canada.

The following data have been presented as a poster in the following peer-reviewed conferences: 2018 International Congress on Palliative Care (PAL), Montreal, Canada (October 2018).
}

*Address correspondence to: Jenny J. Ko, MD, FRCPC, Department of Medical Oncology, University of British Columbia, BC Cancer-Abbotsford, 32900 Marshall Road, Abbotsford, British Columbia V2S OC2, Canada, E-mail: jenny.ko@bccancer.bc.ca

(C) Jenny J. Ko et al., 2020; Published by Mary Ann Liebert, Inc. This Open Access article is distributed under the terms of the Creative Commons License (http://creativecommons.org/licenses/by/4.0), which permits unrestricted use, distribution, and reproduction in any medium, provided the original work is properly cited. 


\section{Background}

Advance care planning (ACP) is "a process that supports adults at any age or stage of health in understanding and sharing their personal values, life goals, and preferences regarding future medical care. The goal of ACP is to help ensure that people receive medical care that is consistent with their values, goals and preferences during serious and chronic illness." Clinicianled ACP discussions about prognosis and patient preferences increase the quality of care perceived by patients and families and may reduce anxiety and depression. ${ }^{2-6}$

In patients with cancer, ACP conversations have been shown to be instrumental in both tailoring management plans that accord with patient values and communicating these plans with physicians and substitute decision makers. ${ }^{7-10}$ The ACP discussions should occur earlier in the cancer trajectory, but the majority still occur close to the end of life. ${ }^{1-13}$ Enhancing the skills of physician learners may provide a critical opportunity to improve the quality and timing of ACP.

Ariadne Labs developed the serious illness care program as a system-level intervention to improve the prevalence, timing, and quality of ACP in serious illness, through implementation of communication skills training and coaching and systems changes that support adoption and use of clinical conversation tools. ${ }^{14}$ The serious illness conversation guide (SICG), the signature clinical tool, comprises a seven-item framework to help clinicians share prognosis and elicit critical information to inform future decision making and care, including illness understanding, decision-making preferences, goals and fears, views on trade-offs and impaired function, and caregiver involvement. ${ }^{15} \mathrm{~A}$ number of health systems in which the current study was situated have adopted the SICG as the basic framework for ACP discussions in provincial cancer clinics.

Recently, the Royal College of Physicians and Surgeons of Canada has initiated restructuring of postgraduate medical training and assessment paradigms with a shift from a time-dependent to a competencybased model. A time-dependent model assumes acquisition of competency with a length of time in training, whereas competency-based medical education (CBME) focuses on outcomes and abilities as the organizing principle of curricular design. To our knowledge, no assessment tool has yet been developed or validated specifically for evaluation of trainee competency in SICs in oncology patients.

\section{Objectives}

The objectives of our study were to develop and validate a novel tool, the SIC-evaluation exercise (SICEx), for assessment of trainee competency in leading SICs with oncology patients in the ambulatory setting. This article presents results from the quantitative analyses; qualitative analyses from the narrative data have been presented as a conference abstract and will be reported in a separate article. ${ }^{16}$

\section{Methods}

SIC-Ex development

The study was approved by the provincial Research Ethics Board. We developed the SIC-Ex tool based on the mini-clinical evaluation exercise (CEx), the palliative care CEx, and SICG framework. ${ }^{17-19}$ The content and format of the checklist items were derived from and formatted based on the domains of conversations described in the SICG, and three steps that Sudore and Fried indicate as important for assessing patients' and surrogates' needs in preparing for in-the-moment decision making. ${ }^{20,21}$

This process has followed the guidelines for developing evaluation checklists published by the Joint Committee on Standards for Educational Evaluation. ${ }^{22}$ A draft of the SIC-Ex was constructed. This draft was reviewed for relevance, comprehensiveness, applicability, and clarity and it was finalized through an iterative process by palliative care physicians and content experts with an interest in medical education and SICs. Oncologists and program directors for academic oncology residency programs also reviewed the SIC-Ex for congruency with CBME models of assessment.

The final version of SIC-Ex covered four domains defined as trainee competency milestones, with specifically assigned questions to which each evaluator could give a numerical score between 1 (needs further instruction) and 4 (competent to perform independently) (Supplementary Appendix SA1):

- Communication basics (professional, communi${\text { cator })^{23}}^{23}$

○ Demonstrated nonverbal empathy. For example, sat down, made eye contact

- Demonstrated verbal empathy. For example, named emotions, understood emotions, stated respect for patient, offered support

- Used open-ended questions

- Introducing ACP (professional, communicator, health advocate) 
- Introduced ACP as a relevant topic for this patient, for example, benefit for patient/family, "Hope for the best, prepare for the worst"

- Clarified components of ACP previously engaged in

- Obtained permission from patient/family to proceed

- Learning about the patient (professional, communicator, leader, scholar)

- Understanding: clarified patient's understanding of illness (including diagnosis, treatments, prognosis)

- Information preferences: assessed patient readiness to engage in ACP conversation. For example, some patients like to know about time, other like to know what to expect, others like to know both, others neither.

- Prognosis: shared prognosis of current illness with patient, tailored to information preferences

- Goals: inquired about patient's own values and health care goals if medical condition worsens.

- Fears/worries: explored patient's fears and/or worries with regard to the future of his/her health.

- Function: explored activities that the patient deems critical to having an acceptable quality of life.

- Trade-offs: explored medical treatments the patient would be willing to go through to gain more time living.

- Family.

$>$ Explored how much the patient's family/friends may know about his/her priorities and wishes

$>$ Determined whether there were other important friends or family members who needed to be included in future ACP conversations

$>$ Asked who the patient would like as a substitute decision maker

- Planning (professional, communicator, leader, collaborator)

- Affirmed commitment to continue caring for patient

$>$ Acknowledged medical realities

$>$ Summarized key goals/priorities

$>$ Described treatment options that reflect goals/priorities

- Made recommendations about the next steps

- Documented conversation

- - Provided patient with written information pertaining to local ACP policies (e.g., ACP conversation guide)
It also included an ordinal global rating ("Overall what is your impression about how this student led the ACP conversation?") capturing the evaluator's impression of the trainee's overall competence (scores $1-4$, where $1=$ very satisfied and $4=$ very dissatisfied).

\section{SIC-Ex validation}

We recruited volunteer trainees and preceptors (evaluators below) from three academic cancer centers. Volunteer patients were recruited from three cancer clinics. All volunteers provided informed consent. Volunteer trainees received a 15-minute introduction to the SICG, but they did not receive standardized training. Each trainee was assigned to a volunteer patient and asked to lead a single SIC in an outpatient oncology clinic. We videotaped each session. Trainees completed a selfassessment survey immediately after the SIC.

After watching the videotapes, evaluators used the SICEx tool (experimental tool) and Calgary-Cambridge guide (CCG; reference tool) to score trainee performance at month 0 (defined as the first time that the evaluator assessed the scores within six months of completing the discussion). The CCG was chosen as a reference tool as it is a validated, widely used, and well-known evaluation tool for trainees to evaluate their communication skills, with relevant domains comparable to the domains in the SIC-Ex-Initiating the session; Gathering information; Providing structure; Building relationship; Explanation and planning; Closing the session; Options in explanation and planning.

The same evaluators watched the videos again three months later and rated them by using the same tools. This article presents results from the quantitative analyses; qualitative analyses from the narrative data have been presented as an abstract and will be reported in a separate article. ${ }^{16}$ All statistical analyses were performed by using the open source software $\mathrm{R}^{24}$ We adopted Kane's framework ${ }^{25,26}$ to generate validity evidence for the SIC-Ex as follows:

(1) Defining the proposed use: SIC may occur within a single discussion or over several discussions at different times and with different health care professionals. For the purpose of the study, all of the domains of the SIC-Ex were evaluated to standardize tool administration. The tool is intended for oncology trainees at all levels of training (e.g., residents, fellows).

(2) Scoring: We defined it as transformation of observation(s) into an insightful and accurate response in the forms of scores and narratives. 
components with their estimated values in the formula:

$$
\frac{\sigma_{\text {evaluator }}^{2}+\sigma_{\text {trainee }}^{2}+\sigma_{\text {evaluator:trainee }}^{2}}{\sigma_{\text {evaluator }}^{2}+\sigma_{\text {trainee }}^{2}+\sigma_{\text {evaluator:trainee }}^{2}+\sigma_{\text {error }}^{2}},
$$

where the variance components correspond to a linear mixed-effects model that treats each type of evaluator score as the outcome variable (measured at month zero and three for each evaluator-trainee combination), while including a fixed-effect intercept and random effects for the evaluator, trainee, and their interaction.

Intra-evaluator reliability of the (ordinal) global rating on SIC-Ex at month zero was calculated separately for each evaluator via Gwet's AC1 and BrennanPrediggers' reliability coefficients, then summarized across evaluators, and finally interpreted based on Altman's benchmark scale. ${ }^{27}$

(4) Extrapolation (Extension of generalized interpretations from the test setting) into real-life situations (i.e., extrapolation beyond the evidence into a new context))

(a) Correlation with CCG: For each evaluator, the correlation between the (quantitative) total scores across all domains of the SIC-Ex and CCG instruments was computed at months zero by performing simple linear regressions involving the two sets of scores and computing Pearson's correlation coefficients; correlation coefficients of 0.4 or higher and ideally larger than 0.6 were desired.

(b) Agreement between trainees and evaluators: Agreement at month zero between trainees and evaluators was assessed by adding the trainee total self-scores on SIC-Ex to the evaluator total scores-separately for each domain and for all domains combined-and computing Krippendorffs $\alpha$. The magnitude of $\alpha$ was interpreted in relation to 0 (perfect disagreement) and 1 (perfect agreement). Further, a decrease in the value of $\alpha$ compared with the one obtained by using only the evaluator ratings was interpreted to indicate that the added self-ratings did not influence inter-evaluator agreement negatively, implying that evaluators agree with trainees. ${ }^{27}$

(5) Implications (Translation of assessment results into meaningful decisions and actions, and the downstream effects of such decisions)

The global ordinal rating and domain scores from the SIC-Ex were provided to each of the trainee tor, trainee, interaction, and error variance 
participants, along with the narrative comments provided by evaluators. Trainees had a de-briefing session with their preceptors after the video recording and completed the Student Post-Encounter Probe (Supplementary Appendix SA2) to determine their perception of: time/difficulty involved in completing the exercise, self-perceived competence in conducting elements of or the whole discussion under observation, and the educational value of the SIC-Ex. The preceptor also recorded his or her own response to two post-intervention questions that asked about the utility and process of the SIC-Ex (see Supplementary Appendix SA2).

\section{Results}

We recruited seven residents and seven patients from two study sites (Abbotsford, Vancouver) at the end of a one-year study period. Ten evaluators from three study sites (Abbotsford, Vancouver, Calgary) completed the first round of resident evaluations by using the SIC-Ex and Cambridge tools at month 0; nine of these completed the second round of resident evaluations at month three. To prevent potential conflict of interest, the three evaluators who recruited students did not evaluate the students they recruited; subsequently, their evaluations were removed from the final statistical analysis. All seven residents provided self-ratings on the SIC-Ex tool immediately after the conversation.
Item analysis

The median score across trainees per item for SIC-Ex was 3.06 (range 2.54-3.32), and for CCG, 3.51 (range 2.73-3.77). The median scores on SIC-Ex were numerically lower than the median scores on Cambridge.

Inter-evaluator reliability of quantitative scores generated by SIC-Ex (i.e., "does one evaluator rate similarly to group average for each domain for the same trainee?")

For the quantitative scores generated by SIC-Ex (i.e., domain-specific scores and total scores across domains), the inter-evaluator reliability at month zero was evaluated by computing intra-class correlation coefficients (ICCs) based on the average of these scores across evaluators for each trainee. The results revealed moderate inter-evaluator reliability at month 0 , whether we measured absolute agreement or consistency. Sensitivity analyses excluding outliers did not significantly impact the results.

Inter-evaluator reliability of global ordinal rating generated by SIC-Ex (i.e., "does one evaluator rate similarly to group average for the global rating for the same trainee?")

For the ordinal global rating generated by SIC-Ex, inter-evaluator reliability at month zero was assessed by computing Brennan-Predigger and Gwet's AC1 reliability coefficients and associated 95\% CIs (Fig. 1).

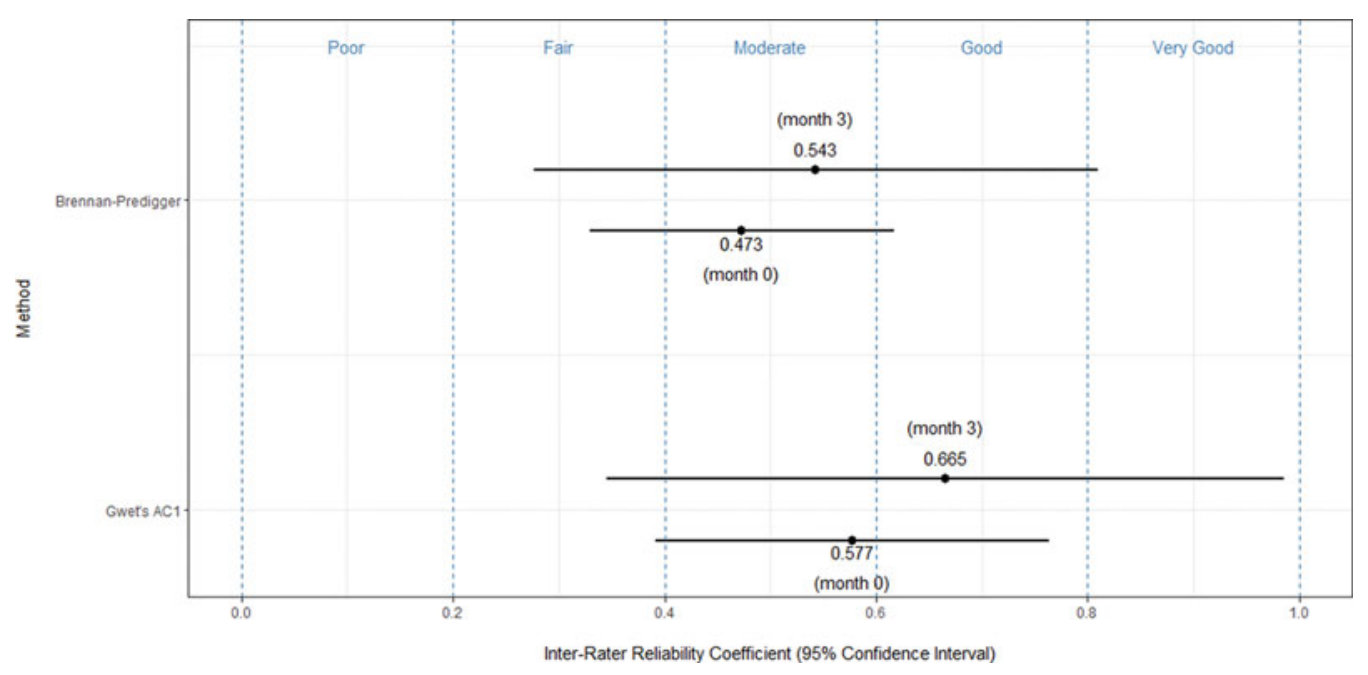

Fig. 1. Inter-evaluator reliability at months zero for the (ordinal) global rating generated by SIC-Ex. SIC-Ex, serious illness conversation-evaluation exercise. 


\section{Month 0}

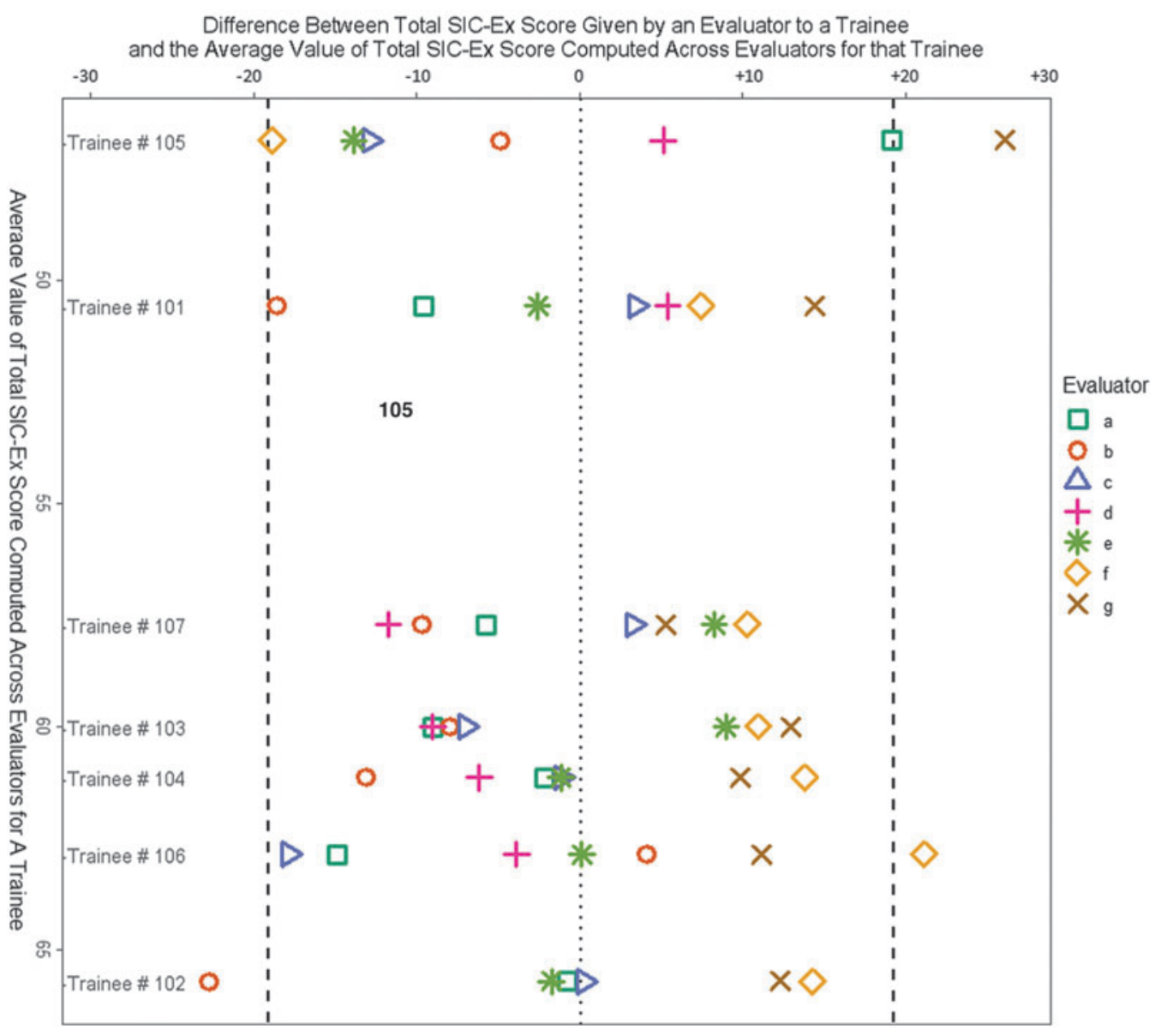

Fig. 2. Modified Bland-Altman plots for the evaluators a-g, derived from the total domain scores across domains on SIC-Ex they assigned to the seven trainees at month zero. If the difference between the total SIC-Ex score given by a particular evaluator to a trainee and the average value of total SIC-Ex score computed across evaluators for that trainee was higher than or lower than \pm 20 (bold dotted vertical lines), the evaluators were considered outliers from the group average. Total SIC-Ex score refers to the total score across all domains of SIC-Ex.

Both tests were performed and coefficients were computed to ensure that the results were comparable. The true inter-evaluator reliability was estimated as fair to good at month zero.

\section{Evaluator agreement with group average based} on quantitative scores generated by SIC-EX

Figure 2 describes the modified Bland-Altman agreement analysis of total scores across domains on SIC-Ex at month zero and shows that most evaluators scored a particular trainee similarly to the group average, except for one or two evaluator outliers who rated the same trainee significantly higher or lower than the group average. Analogous analyses for domain-specific scores on SIC-Ex at month zero revealed comparable findings (not shown).

Intra-evaluator reliability of quantitative and qualitative scores generated by SIC-EX (i.e., does an evaluator rate similarly to oneself for the same trainee between two timepoints?) Intra-evaluator (test-retest) reliability of domain-specific and total scores across domains on SIC-Ex showed a moderate level of self-consistency of evaluators over 
Table 1. Average Score Intra-class Correlation Coefficient Estimates and 95\% Confidence Intervals for Specific Domains of Serious Illness Conversation-Evaluation Exercise and for All Domains Combined, Computed at Month Zero

\begin{tabular}{lcc}
\hline & \multicolumn{2}{c}{$\begin{array}{c}\text { ICC estimate at month } 0 \\
(95 \% \text { confidence interval) }\end{array}$} \\
\cline { 2 - 3 } & Agreement $(n=7)$ & Consistency $(n=7)$ \\
\hline Domain & $0.822(0.531$ to 0.964$)$ & $0.856(0.599$ to 0.971$)$ \\
\hline $\begin{array}{c}\text { "Communication } \\
\text { basics" }\end{array}$ & & \\
$\begin{array}{c}\text { "Introducing ACP" } \\
\text { "Learning about } \\
\text { the patient" }\end{array}$ & $0.523(-0.017$ to 0.888$)$ & $0.666(0.070$ to 0.934$)$ \\
$\begin{array}{c}\text { "Planning" } \\
\text { All domains } \\
\text { combined }\end{array}$ & $0.596(-0.069$ to 0.894$)$ & $0.618(-0.065$ to 0.924$)$ \\
\hline
\end{tabular}

$A C P$, advance care planning; ICC, intraclass correlation coefficient.

time (intra-evaluator reliability ranged from 0.477 to 0.763 for domain-specific scores and was equal to 0.795 for the total score across domains) (Table 1). Intra-evaluator reliability of the (ordinal) global rating on SIC-Ex exhibited a "fair to good" level across evaluators (Table 2).

Correlation with CCG (i.e., does SIC-Ex rate

a trainee similarly as well as CCG?)

For most evaluators, total scores across domains produced by the two tools, SIC-Ex and CCG, were highly correlated at month 0 (Fig. 3). The two outlying evaluators $\mathrm{d}$ and $\mathrm{g}$ with low correlations of 0.28 and 0.37 , respectively, at month zero were also outliers in regards to the inter-evaluator reliability at month 0 .

Agreement between trainees and evaluators based on quantitative scores generated by SIC-Ex (i.e., do trainees' self-scores on SIC-Ex match with those of the evaluators?)

The addition of trainee self-scores to the evaluator scores when computing Krippendorff's $\alpha$ at month zero did not influence inter-evaluator agreement negatively for the "Introducing ACP," "Learning about the patient," "Planning" domains, and for all domains

Table 2. Intra-Evaluator Reliability of Domain-Specific and Total Scores Across Domains Generated by Serious Illness Conversation-Evaluation Exercise

\begin{tabular}{lc}
\hline Domain & Intra-evaluator reliability at month 0 (?) \\
\hline Communication basics & 0.477 \\
Introducing ACP & 0.661 \\
Learning about the patient & 0.763 \\
Planning & 0.692 \\
All domains combined & 0.795 \\
\hline
\end{tabular}

combined, but it did negatively affect the "Communications basics" domain (Table 3). Although the agreement between trainees and evaluators was similar in magnitude to that between evaluators alone, it was on the low or very low side (Table 4).

\section{Discussion}

To the authors' knowledge, our study is the first to demonstrate validity evidence of using SIC-Ex for resident trainees interviewing outpatient cancer patients. It brings together the Serious Illness Conversation framework in the context of competency-based medical education (CBME) evaluation and to validate domain-specific, total domain, and global scores and narrative comments. CBME is "an approach to preparing physicians for practice that is fundamentally oriented to graduate outcome abilities and organized around competencies derived from an analysis of societal and patient needs." ${ }^{29,30}$ The Medical Oncology Subspecialty at the Royal College of Physicians and Surgeons of Canada defines "discussing serious news" and "transitioning away from active anti-cancer therapy" as professional activities that can be entrusted to trainees once their component core competencies have been achieved. The SIC-Ex was constructed around these competencies. As SIC-Ex is a performance-based, formative evaluation process composed of multiple key milestones and integrating multiple domains of competencies, the outcomes (competencies) are not isolated elements of knowledge or a skill, but rather are integrated and observed/measured to ensure their acquisition. Our study demonstrates that it is feasible to assess a trainee's competence by incorporating elements of a pre-existing evidence-based communication tool.

At least three studies have quantified and scored trainees' communication skills in end-of-life discussions or ACP by using non-validated internal scales, although these were based on self-assessment, which is generally a less accurate method of competency assessment, rather than preceptor observations. ${ }^{31-33}$ Previously validated assessment tools in medical communication skills include the CCG and mini$\mathrm{CEx}^{18,19,34}$; however, they are generic tools for assessment of communication skills and are not validated to assess ACP conversation skills. Han et al. previously reported the feasibility and potential effectiveness of the palliative care CEx, a modified version of CEx, to assess trainees' ability to discuss end-of-life issues with patients; again, this tool is not specifically designed to assess ACP conversation skills. ${ }^{17}$ Another 

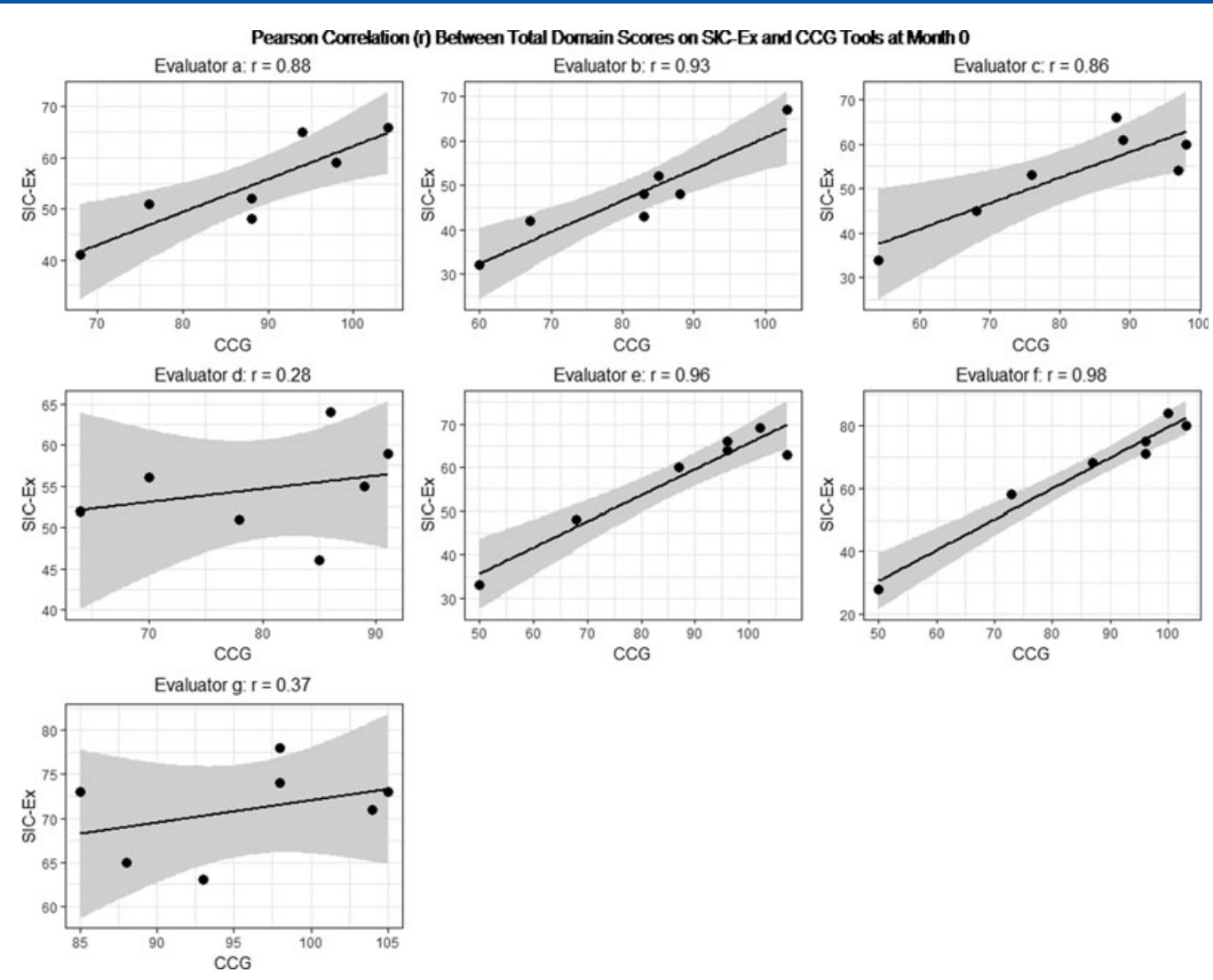

Fig. 3. Correlation between total domain-specific scores and total scores across domains on the SIC-Ex and CCG tools at month zero. CCG, Calgary-Cambridge guide.

recent study developed and validated an instrument, ACP-Communication Assessment Tool (CAT), to assess ACP communication skills of clinicians and trainees. ${ }^{35}$ The ACP-CAT has domains that overlap with the SIC framework; however, the participating trainees did not have formal orientation to the SIC, and the conversations were done in the context of an examination with a simulated patient. Our study allows an incorporation of an evaluation instrument for SIC into the CBME for assessment of trainees in a real-world outpatient clinic setting.

Table 3. Intra-Evaluator Reliability of Ordinal Global Rating Generated by Serious IIIness Conversation-Evaluation Exercise, Summarized across Evaluators

\begin{tabular}{lccc}
\hline $\begin{array}{l}\text { Intra-evaluator } \\
\text { reliability } \\
\text { coefficient }\end{array}$ & $\begin{array}{c}\text { Average } \\
\text { across } \\
\text { evaluators }\end{array}$ & $\begin{array}{c}\text { Standard } \\
\text { deviation across } \\
\text { evaluators }\end{array}$ & $\begin{array}{c}\text { Range across } \\
\text { evaluators } \\
\text { (minimum to } \\
\text { maximum) }\end{array}$ \\
\hline $\begin{array}{l}\text { Gwet's AC1 } \\
\text { Brennan-Predigger }\end{array}$ & 0.762 & 0.138 & $0.586-0.906$ \\
\hline
\end{tabular}

Overall, our study demonstrated that the SIC-Ex scores for most of the domains were lower with more discriminatory variations among residents than those of the CCG for each resident. This may indicate an increased specificity of SIC-ex scores for the particular

Table 4. Krippendorff's $\alpha$ Values at Month Zero Derived from (a) Evaluator Scores on Serious Illness ConversationEvaluation Exercise (SIC-Ex) and (b) Evaluator Scores Plus Trainee Self-Scores on SIC-Ex. Both Domain-Specific Scores and Scores Across All Domains Were Considered for Trainees and Evaluators

\begin{tabular}{lcc}
\hline & \multicolumn{2}{c}{ Krippendorff's $\alpha$ at month 0} \\
\cline { 2 - 3 } Domain & $\begin{array}{c}\text { (A) Evaluator } \\
\text { scores on } \\
\text { SIC-EX }\end{array}$ & $\begin{array}{c}\text { (B) Evaluator scores } \\
\text { plus trainee self-scores } \\
\text { on SIC-EX }\end{array}$ \\
\hline Communication basics & 0.323 & 0.304 \\
Introducing ACP & 0.0895 & 0.103 \\
Learning about the patient & 0.102 & 0.102 \\
Planning & 0.0559 & 0.0854 \\
All domains combined & 0.135 & 0.141 \\
\hline \multicolumn{2}{c}{ SIC-Ex, serious illness conversation-evaluation exercise. }
\end{tabular}


domains assessed by each question compared with more generic communications assessed by CCG. Literature suggests that generic evaluation scores for communication skills are not necessarily formative, as they may provide feedback that is often too general and not specific to the skillsets required for a certain milestone. ${ }^{36,37}$ Generic evaluation scores may not generate recommendations on how a learner can improve and build on the specific skill sets. Studies also suggest that generic evaluation scores may need to be tailored to the specific student group. ${ }^{38}$ This same evidence suggests that more tailored evaluation scores tend to improve the training group performance over time. In addition, the narrative data generated from the written comments by the evaluators are critical to the formative nature of the evaluation; this has been presented as a conference abstract ${ }^{16}$ and will be explored in a separate article. The SIC-Ex allows for the written comments in each domain and also for a global summary.

We observed overall high inter- and intra-evaluator variabilities. Evaluators had no formal training and did not undertake normative processes for evaluating residents using the SIC-Ex and CCG. Training and orientation on the use of SIC may reduce inter- and intraevaluator variability. Although all of our evaluators were familiar with SIC, some may have been more comfortable with its use than others given their practice patterns. Another aspect is familiarity with the formative evaluation process itself. There are data to suggest that faculty development on use of evaluative tools and feedback mechanisms is essential to training evaluators to implement a successful mini-CEx assessment program. ${ }^{39,40}$ Guidance for evaluators on the CEx as a tool to foster the preceptor - student relationship may improve the effectiveness of the SIC-Ex process.

Trainee self-scores did not correlate well with evaluator scores. Other studies show poor correlation between trainees' self-assessment and preceptor, patient, or family reviews of a trainee's performance, particularly in communication competencies and outcomes of end-of-life care. ${ }^{41-43}$ Literature suggests that experiential training as well as a validated evaluative tool for formative feedback may lessen the gap between the self-assessment of trainees and evaluator/patient/ family assessments. ${ }^{43}$ Given the importance of serious illness discussions in patients with cancer, a tool such as the SIC-Ex could help with a formative educational process in this area.

Our study shows that SIC-Ex can be used for the following purposes: (1) ongoing assessments of resi- dent trainees in the outpatient oncology clinic settings to evaluate the CBME-based milestones, with comprehensive periodic reviews to ensure continued progress, as a trainee may receive both quantitative and qualitative feedback over a period of time to document progress in each domain and globally. However, we acknowledge that in this study, the residents were only assessed at one time point with no reassessment; (2) use of multiple assessors and assessments to enable the right assessment to be made at the right time for the right purpose, as the behavioral attributes evaluated through SIC-Ex are comprehensive and incorporate both generic attributes and those specific to serious illness conversations; (3) mechanisms to synthesize data collected through group processes to reach judgments about competence (e.g., as an Entrustable Physician Activity), as inter-evaluator reliability, evaluator agreement with the group mean, and intraevaluator reliability are at least fair to good; (4) faculty development for all assessors, as it is critical for the evaluators to be familiar with the SICG; and (5) optimized relationships between the givers and receivers of formative feedback to enhance the incorporation of feedback into practice. ${ }^{44}$

The limitations of our study include the following: Due to small sample size, the generalizability of the study findings needs to be tested in a broader context. The current study did not test whether the SIC-Ex scores are responsive and sensitive to changes in the trainees' SIC skills. This will need to be examined in larger future studies. The discrepancy between trainees' self-scores and scores by evaluators (preceptors) may reflect a need to provide additional learner-centered instructions on use and evaluation of SIC to evaluators and trainees. The findings need to be taken in the context of small sample size and limited evaluator and/or trainee training on SIC before the study. Despite the validity evidence supporting use of SIC-Ex, the core Medical Oncology competencies were not developed around the SIC framework and therefore a broader use of SIC in oncology residencies needs to be examined in the current context of predefined milestones. In our statistical analysis, we used unweighted sums to compute the domain-specific scores and the total scores across all four domains of SIC-Ex. This assumes that all items of a domain are equally important in the determination of a domain-specific score, and that all items of SIC-Ex are equally important in the determination of the total score across domains. Due to the small sample size, and the fact that the items are 
extracted directly from the already established SIC framework, we did not further test each item or domain against the others in this study. Lastly, there may be possible selection bias in using tools on volunteer patients and volunteer learners.

In conclusion, this study adds validity evidence to support use of SIC-Ex in assessing resident competencies in serious illness communication. Use of the SICEx has the potential to enhance formative evaluation and feedback process for residents having serious illness conversation with oncology patients. Further resident and evaluator training on SIC-Ex may enhance its reliability in evaluating domains that are specific to serious illness conversations.

\section{Funding Information}

This study was funded by Medical Council of Canada Research in Clinical Assessment Grant.

\section{Author Disclosure Statement}

No competing financial interests exist.

\section{Supplementary Material \\ Supplementary Appendix SA1 \\ Supplementary Appendix SA2}

\section{References}

1. Sudore RL, Lum HD, You JJ, et al.: Defining advance care planning for adults: A consensus definition from a multidisciplinary Delphi panel. J Pain Symptom Manage 2017;53:821.e821-832.e821.

2. Heyland DK, Allan DE, Rocker G, et al.: Discussing prognosis with patients and their families near the end of life: Impact on satisfaction with end-oflife care. Open Med 2009;3:e101-e110.

3. Heyland DK, Cook DJ, Rocker GM, et al.: Defining priorities for improving end-of-life care in Canada. CMAJ 2010;182:E747-E752.

4. Wright $A A$, Zhang $B$, Ray $A$, et al.: Associations between end-of-life discussions, patient mental health, medical care near death, and caregiver bereavement adjustment. JAMA 2008;300:1665-1673.

5. Smucker WD, Ditto PH, Moore KA, et al.: Elderly outpatients respond favorably to a physician-initiated advance directive discussion. J Am Board Family Pract 1993;6:473-482.

6. Paladino J, Bernacki R, Neville BA, et al.: Evaluating an intervention to improve communication between oncology clinicians and patients with life-limiting cancer: A cluster randomized clinical trial of the serious illness care program. JAMA Oncol 2019;5:801-809.

7. McCarthy EP, Phillips RS, Zhong Z, et al.: Dying with cancer: Patients' function, symptoms, and care preferences as death approaches. J Am Geriatr Soc 2000;48(5 Suppl.):S110-S121.

8. Lynn J, Teno JM, Phillips RS, et al.: Perceptions by family members of the dying experience of older and seriously ill patients. SUPPORT Investigators. Study to understand prognoses and preferences for outcomes and risks of treatments. Ann Intern Med 1997;126:97-106.

9. Earle CC, Neville BA, Landrum MB, et al.: Trends in the aggressiveness of cancer care near the end of life. J Clin Oncol 2004;22:315321.

10. Earle CC, Landrum MB, Souza JM, et al.: Aggressiveness of cancer care near the end of life: Is it a quality-of-care issue? J Clin Oncol 2008;26: 3860-3866.
11. Lopez-Acevedo M, Havrilesky $\sqcup$, Broadwater G, et al.: Timing of end-oflife care discussion with performance on end-of-life quality indicators in ovarian cancer. Gynecol Oncol 2013;130:156-161.

12. Mack JW, Cronin A, Taback N, et al.: End-of-life care discussions among patients with advanced cancer: A cohort study. Ann Intern Med 2012;156: 204-210.

13. El-Sahwi KS, Illuzzi J, Varughese J, et al.: A survey of gynecologic oncologists regarding the end-of-life discussion: A pilot study. Gynecol Oncol 2012;124:471-473.

14. Bernacki R, Hutchings $M$, Vick J, et al.: Development of the serious illness care program: A randomised controlled trial of a palliative care communication intervention. BMJ Open 2015;5:e009032.

15. Bernacki RE, Block SD; American College of Physicians High Value Care Task F: Communication about serious illness care goals: A review and synthesis of best practices. JAMA Intern Med 2014;174:1994-2003.

16. Ko J, Ballard MS, Roze des Ordons A, Shenkier T, et al.: Validation of a novel assessment tool to evaluate how well medical residents are prepared to lead Serious IIIness Conversations with oncology patients based on the competency-based medical education (CBME) model-A qualitative analysis. In: Paper presented at: 018 International Association for Medical Education (AMEE) Annual Meeting; August 26, 2018, 2018; Basel, Switzerland.

17. Han PK, Keranen LB, Lescisin DA, Arnold RM: The palliative care clinical evaluation exercise (CEX): An experience-based intervention for teaching end-of-life communication skills. Acad Med 2005;80:669-676.

18. Simmenroth-Nayda A, Heinemann S, Nolte C, et al.: Psychometric properties of the Calgary Cambridge guides to assess communication skills of undergraduate medical students. Int J Med Educ 2014;5:212-218.

19. Norcini JJ, Blank LL, Duffy FD, Fortna GS: The mini-CEX: A method for assessing clinical skills. Ann Intern Med 2003;138:476-481.

20. Speak Up-Start the conversation about end-of-life care: www .advancecareplanning.ca/about-advance-care-planning.aspx. 2014. (Last accessed April 1, 2020).

21. Sudore RL, Fried TR: Redefining the "planning" in advance care planning: Preparing for end-of-life decision making. Ann Intern Med 2010;153: 256-261.

22. Stufflebeam DL: The checklists development checklist. Western Michigan University Evaluation Center: www.wmich.edu/evalctr/checklists/cdc .htm. 2000. (Last accessed September 4, 2014).

23. Frank JSL, Sherbino J: CanMEDS 2015 Physician Competency Framework: www.royalcollege.ca/rcsite/documents/canmeds/canmeds-fullframework-e.pdf. 2015. (Last accessed August 23, 2019).

24. Fried TR, Redding CA, Robbins ML, et al.: Stages of change for the component behaviors of advance care planning. J Am Geriatr Soc 2010; 58:2329-2336.

25. Cook DA, Brydges R, Ginsburg S, Hatala R: A contemporary approach to validity arguments: A practical guide to Kane's framework. Med Educ 2015;49:560-575.

26. Hatala R, Sawatsky AP, Dudek N, et al.: Using in-training evaluation report (ITER) qualitative comments to assess medical students and residents: A systematic review. Acad Med 2017;92:868-879.

27. Gwet K: Handbook of Inter-Rater Reliability: The Definitive Guide to Measuring the Extent of Agreement Among Raters, 4th ed. Gaithersburg, MD: Advanced Analytics LLC, 2014.

28. Jones M, Dobson A, O'Brian S: A graphical method for assessing agreement with the mean between multiple observers using continuous measures. Int J Epidemiol 2011;40:1308-1313.

29. Competence by Design (CBD): Moving toward competency-based medical education: www.royalcollege.ca/portal/page/portal/rc/resources/ cbme. 2015. (Last accessed January 19, 2016).

30. Frank JRMR, Ahmad Y, Wang M, et al.: Toward a definition of competencybased education in medicine: A systematic review of published definitions. Med Teacher 2010;32:631-637.

31. Eggly S, Afonso N, Rojas G, et al.: An assessment of residents' competence in the delivery of bad news to patients. Acad Med 1997;72:397-399.

32. Alderman JS, Nair B, Fox MD: Residency training in advance care planning: Can it be done in the outpatient clinic? Am J Hosp Palliat Care 2008; 25:190-194.

33. Alexander SC, Keitz SA, Sloane R, Tulsky JA: A controlled trial of a short course to improve residents' communication with patients at the end of life. Acad Med 2006;81:1008-1012. 
34. Kurtz SSJ, Draper J: Teaching and Learning Communication Skills in Medicine, 2nd ed. Oxford: Radcliffe, 2005.

35. Yuen JK, Kelley AS, Gelfman LP, et al.: Development and validation of the ACP-CAT for assessing the quality of advance care planning communication. J Pain Symptom Manage 2020;59:1.e3-8.e3.

36. Soemantri D, Dodds A, McColl G: Examining the nature of feedback within the Mini Clinical Evaluation Exercise (Mini-CEX): An analysis of 1427 MiniCEX assessment forms. GMS J Med Educ 2018;35:Doc47.

37. Berendonk C, Rogausch A, Gemperli A, Himmel W: Variability and dimensionality of students' and supervisors' mini-CEX scores in undergraduate medical clerkships - a multilevel factor analysis. BMC Med Educ 2018;18:100.

38. Liu YP, Jensen D, Chan CY, et al.: Development of a nursing-specific MiniCEX and evaluation of the core competencies of new nurses in postgraduate year training programs in Taiwan. BMC Med Educ 2019;19:270

39. Liao KC, Pu SJ, Liu MS, et al.: Development and implementation of a miniClinical Evaluation Exercise (mini-CEX) program to assess the clinical competencies of internal medicine residents: From faculty development to curriculum evaluation. BMC Med Educ 2013;13:31.

40. Hashim M, Prinsloo A, Leduc C, et al.: What community-based preceptors want in teaching medical students: Findings from a mixed methods study. Educ Health 2010;23:366.

41. Dickson RP, Engelberg RA, Back AL, et al.: Internal medicine trainee selfassessments of end-of-life communication skills do not predict assessments of patients, families, or clinician-evaluators. J Palliat Med 2012;15: 418-426.

42. Amery J, Lapwood S: A study into the educational needs of children's hospice doctors: A descriptive quantitative and qualitative survey. Palliat Med 2004;18:727-733.
43. Buss MK, Alexander GC, Switzer GE, Arnold RM: Assessing competence of residents to discuss end-of-life issues. J Palliat Med 2005;8:363-371.

44. Lockyer J, Carraccio C, Chan MK, et al.: Core principles of assessment in competency-based medical education. Med Teacher 2017;39:609-616.

Cite this article as: Ko JJ, Ballard MS, Shenkier T, Simon J, Roze des Ordons A, Fyles G, Lefresne S, Hawley P, Chen C, McKenzie M, Ghement I, Sanders JJ, Bernacki R, and Jones S (2020) Serious illness conversation-evaluation exercise: A novel assessment tool for residents leading serious illness conversations, Palliative Medicine Reports 1:1, 280-290, DOI: 10.1089/pmr.2020.0086.

\section{Publish in Palliative Medicine Reports}

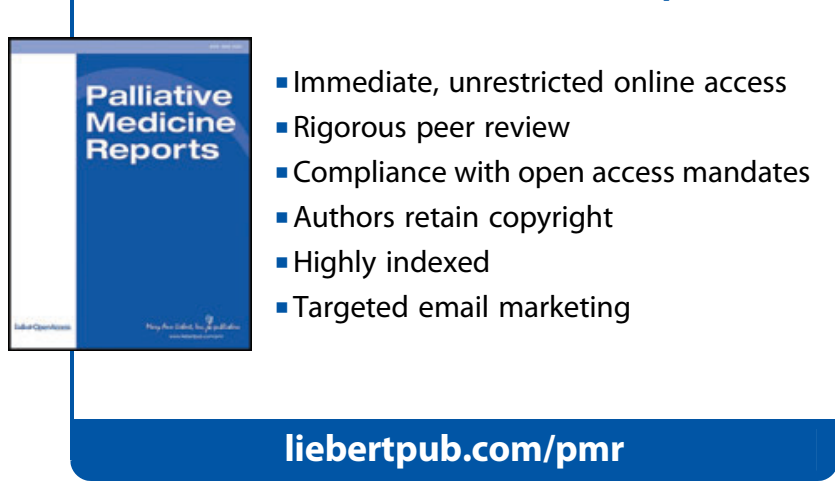

